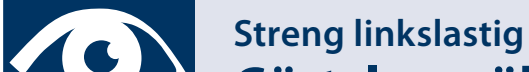

\section{Gürtelrose überraschte mit Blutblasen}

Die Milz war dem 61-Jährigen bereits vor sieben Jahren entfernt worden. Die hartnäckige Thrombozytopenie verschwand aber nicht und trotz Prednisolon sank seine Thrombozytenzahl weiter. Auf die Erhöhung der Dosis reagierte der Mann eigenartig - unzählige Blutblasen bildeten sich an seinem Körper.

Zur Behandlung seiner chronischen Idiopathischen Thrombozytopenie (ITP, Morbus Werlhof) hatte der 61-jährige Mann bereits eine Splenektomie hinter sich und bekam eine medikamentöse Langzeittherapie mit Prednisolon. Da seine Blut-
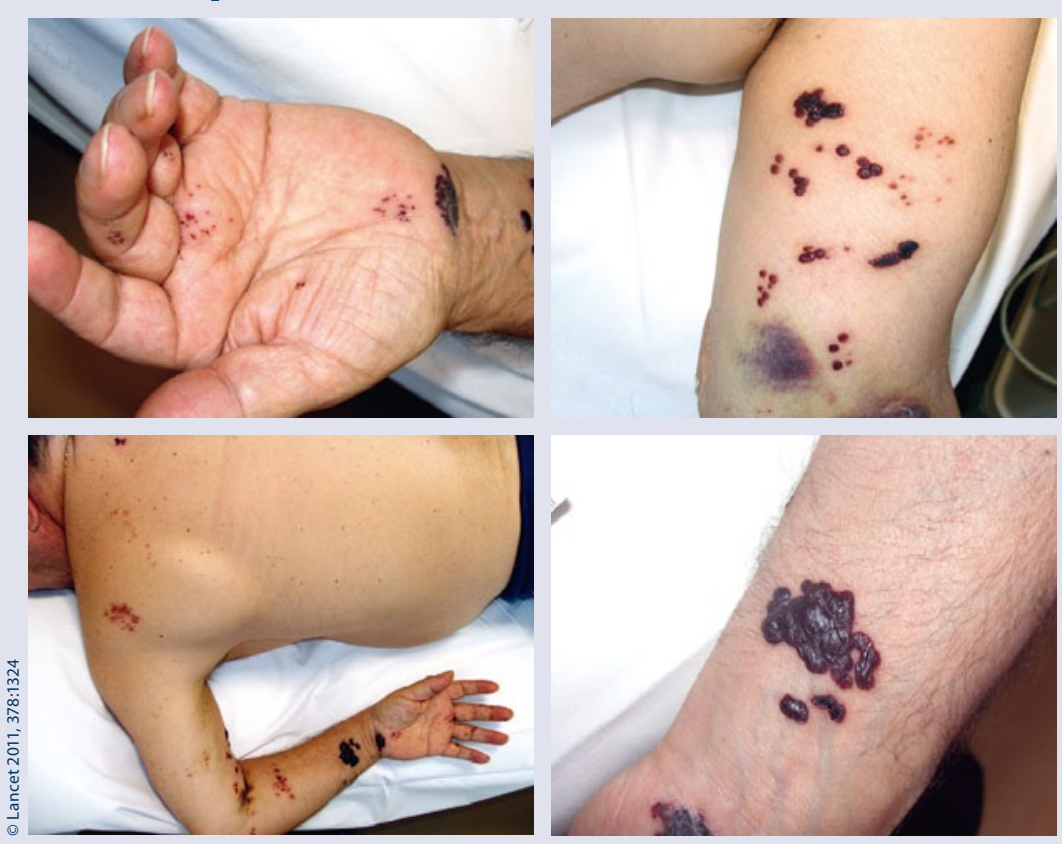

plättchenzahl stark abfiel (6000/ $\mu$ l), erhöhte man die Dosis. 24h später entwickelte der Mann zahlreiche dunkel pigmentierte schmerzhafte Blasen, streng begrenzt auf die linke Seite seines Rumpfes, den linken Arm und die linke Hand. Diese auffällige Verteilung erleichterte die Diagnose: hämorrhagische Herpes-zoster-Infektion, eine atypische Variante der Gürtelrose, die oft im Zusammenhang mit Immunsuppression, Koagulopathien oder Thrombozytopenie in Erscheinung tritt. Die Ärzte stoppten die Prednisolon-Therapie und gaben stattdessen Immunoglobulin $\mathrm{G}$ i. v. ( $\mathrm{g} / \mathrm{kg}$ täglich) und Valaciclovir, eine Vorstufe von Aciclovir (1g dreimal täglich). Die Thrombozytenzahl stieg schnell wieder an und der Patient erholte sich ohne weitere Zwischenfälle.

Lancet 2011, 378:1324

Blutige Blasen sprossen auf der linken Seite des Mannes.

\section{(c) Hingucker aus Praxis und Klinik}

Meist geht es in der Hausarztpraxis ja eher um Schnupfen, Rückenschmerzen oder Hypertonus. Aber bestimmt läuft Ihnen auch mal der eine oder andere Patient mit einem optisch außergewöhnlichen Befund ins Haus!
Machen Sie mit - schicken Sie uns einen spannenden Fall, denn wir möchten interessante Bilder aus Klinik, Praxis und unserem großen Springer-Archiv aufgreifen und in jedem CME einen „Hingucker“ bringen. Für jeden ver- öffentlichten Befund gibt es ein spannendes Springer Lehrbuch. Schicken Sie Ihren Fall an: Springer Medizin Verlag, Frau Claudia Daniels, Tiergartenstr. 17, 69121 Heidelberg, claudia.daniels@springer.com. beiden Zulassungsstudien mehr Probanden in der Febuxostat- (26-39\%) als in der Allopurinol-Gruppe (21-26\%) die Therapie vorzeitig abbrachen.

Unter Febuxostat steigt außerdem das kardiovaskuläre Risiko, zumindest zeichnete sich das in den beiden Zulassungsstudien wie auch in beiden Verlängerungsstudien $\mathrm{ab}$. Ein wichtiger Punkt, der bei der Verordnung unbedingt berücksichtigt werden muss. Auch wenn ein kausaler Zusammenhang nicht direkt belegt werden kann, wird in den Fachinformationen Febuxostat (Stand Dez. 2009) dringend davon abgeraten,
Patienten mit koronarer Herzkrankheit oder Herzinsuffizienz mit Febuxostat zu behandeln.

\section{Das råt der Experte!}

Für den Göttinger Pharmakologen ist und bleibt Allopurinol der „Goldstandard“ in der Gichtanfallprophylaxe. Bei Patienten mit einer Allopurinolunverträglichkeit, eingeschränkter Nierenfunktion oder Polymedikation mit dem Risiko klinisch relevanter Arzneimittelinteraktionen sei Febuxostat aber eine wirksame Alternative. Besonde- re Vorsicht sei jedoch bei Patienten mit kardiovaskulären Vorerkrankungen geboten. Auch die zehnfach höheren Tagestherapiekosten - 40 Euro für 80 mg Febuxostat versus 0,14 Euro für 300 mg Allopurinol - müssen in die Therapieentscheidung einfließen. Um jedoch endgültige Aussagen zu Therapietreue, Langzeittoxizität und -effektivität treffen zu können, sei es noch zu früh. Das müsse erst in großen Vergleichsstudien untersucht werden. (Dagmar Kraus)

El-Armouche A et al. Febuxostat, ein neues Pharmakon zur Behandlung der Gicht. Kardiologe 2011; 5: 45-50 